\title{
The effects of ligand charge, orientation and size on the binding of potential inhibitors for Aldehyde Dehydrogenase
}

Caroline A. Magee, Larryn W. Peterson and Mauricio Cafiero

Department of Chemistry, Rhodes College, 2000 North Parkway Memphis, TN 38112, USA

\begin{abstract}
:
L-DOPA, used as a therapy for patients with Parkinson's disease, is transformed into needed dopamine in the brain. This dopamine can then be deactivated via metabolism by a series of enzymes, including aldehyde dehydrogenase (ALDH). The targeted inhibition of the ALDH enzyme may help to prolong LDOPA therapy. A series of potential inhibitors has been studied via ab initio models using a crystalstructure of the ALDH enzyme with an inhibitor bound in its active site (PDB ID: 4WP7). The positions of novel dopaminergic derivatives were optimized in the active site using M062X/6-31G with implicit solvation and relaxed amino-acid side-chains. This work examines different single molecule orientations, as well as double molecule configurations. Various sizes of ligands were also studied. Interaction energies between the ligands and the protein were calculated using M062X with the $6-311+G *$ basis set. Some potential inhibitors show promising results such as the MP and CAM series.
\end{abstract}

\section{Introduction:}

Aldehyde dehydrogenase (ALDH) plays an important role in the metabolism of dopamine. Many patients that have Parkinson's disease (PD) have low levels of dopamine in their brain due to the degradation of the substantia nigra, where much of the production of dopamine takes place ${ }^{1}$. Dopamine replacement derived from L-DOPA is commonly used as a treatment for patients with Parkinson's disease. L-DOPA can be metabolized or transformed before or after passing through the blood brain barrier (BBB), thereby decreasing the amount of dopamine that can be produced ${ }^{2}$. The targeted, reversible inhibition of the ALDH enzyme may help to prolong the effectiveness of L-DOPA, resulting in a net increase in pharmacological efficiency for L-DOPA. This work and related work in our lab has the 
goal of finding a drug candidate that will inhibit enzymes in the dopamine pathway, such as aldehyde dehydrogenase (ALDH), while keeping the enzymes in the dopamine synthesis pathway active $e^{3,4,5}$.

Inhibition of ALDH is an attractive target for several conditions and must thus be highly selective and targeted. One specific role of ALDH is as follows (see Fig. 1.); dopamine is oxidized by monoamine oxidase (MAO) to form 3,4-dihydroxyphenylacetaldehyde (DOPAL), which is further oxidized to 3,4dihydroxyphenylacetic acid by the enzyme ALDH ${ }^{6}$. While the current work focuses on Parkinson's disease, targeting this enzyme can also provide treatment for other disorders ${ }^{7}$.

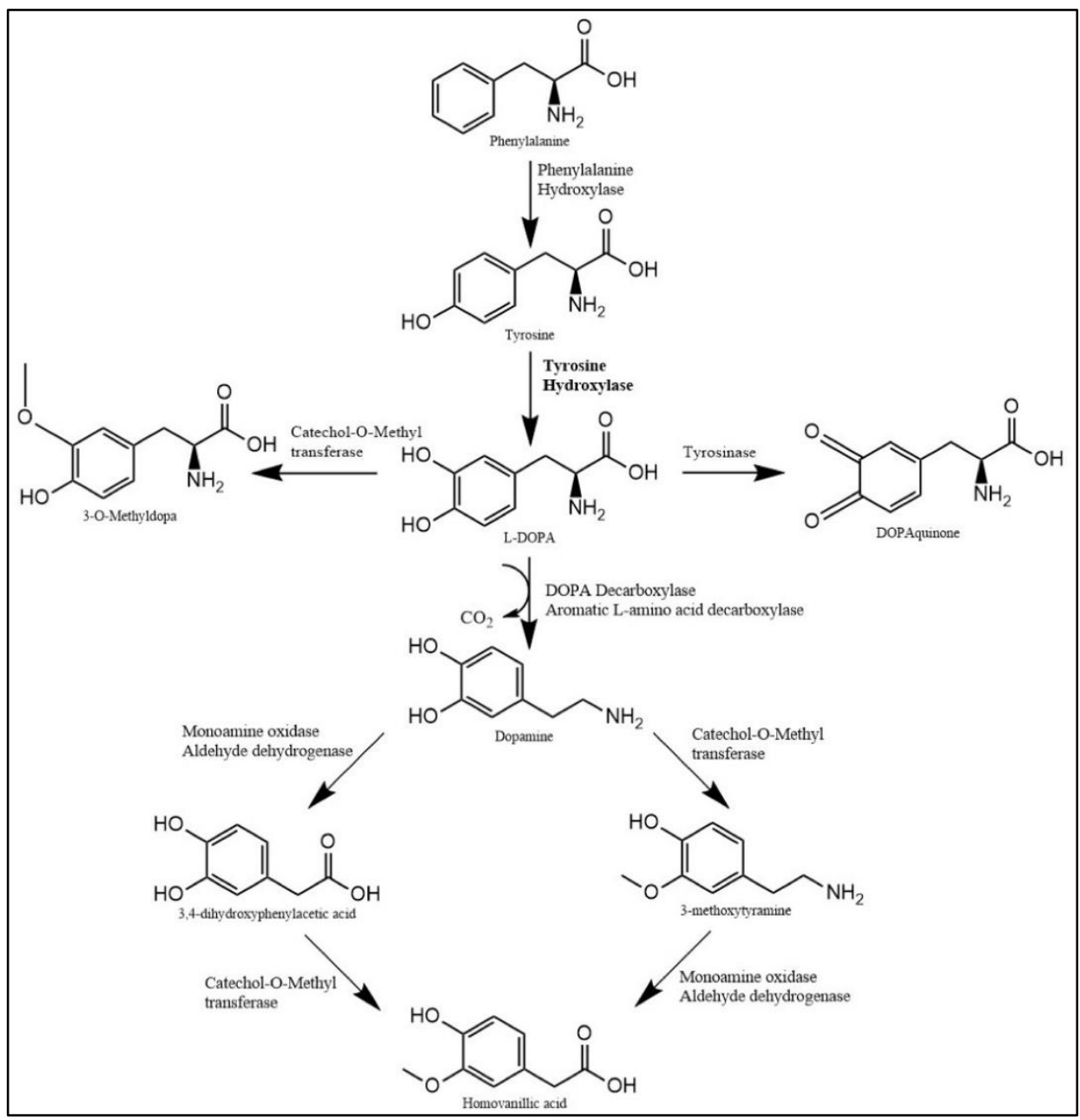

Figure 1. Biosynthetic and degradation pathways of dopamine. 
In this work, a variety of dopaminergic ligands were tested in ALDH to find a potential drug candidate for PD and other disorders. In order to create a smaller library of compounds to test experimentally, computational studies were undertaken to find the interaction energies of potential inhibitors with the active site of ALDH. These potential inhibitors consisted of dopaminergic derivatives that varied in size, charge and substituents. The dopaminergic derivatives were placed in the active site and their positions were optimized using M062X, ${ }^{3,7,8}$ a DFT method that provides accurate energies for non-bonded interactions ${ }^{9}$. M062X was also used for all interaction energy calculations, as previous work has shown that it is among the most accurate DFT methods calculating non-covalent interaction energies $^{3}$. The un-modified M062X functional takes into account weak forces, such as induction and dispersion (see the work of Zhao and Trulhar ${ }^{10,11}$ ) and has been tested against training sets that include weak non-covalent interactions; it does not require additional dispersion, as is shown in our own previous work $^{3-5}$. The current work is based on the differential positioning of ligands in the active site and comparison with the calculated interaction energies of previously tested inhibitors CM026, CM053, and $\mathrm{CM} 037$ (Fig. 2). Upon starting this work, we found the interaction energies within the active site for CM026, CM053, and CM037 to be $-76.94 \mathrm{kcal} / \mathrm{mol},-52.13 \mathrm{kcal} / \mathrm{mol}$, and $-22.38 \mathrm{kcal} / \mathrm{mol}$, respectively ${ }^{10}$. CM026 and CM053 both have good potency for ALDH1A1, but both have bulky R-groups on their xanthine rings, and in the case of $\mathrm{CM} 037$, the xanthine ring is replaced by a tricyclic ring, which also has a long chain substituent ${ }^{8}$. The interactions of these inhibitors with the active site were largely dominated by Gly458 and Trp $178^{8}$. 


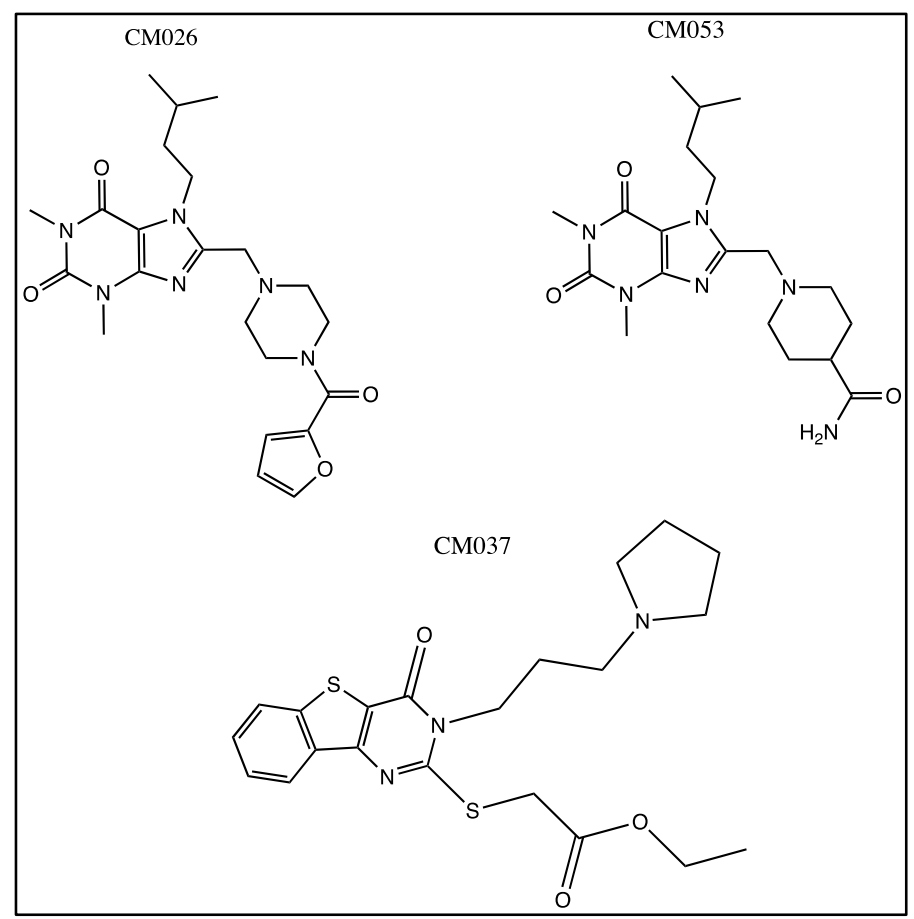

Figure 2. The structures of previously studied inhibitors for ALDH.

ALDH has also been tested with Garcinia bioflavonoids as potential inhibitors towards preventing ALDH pathophysiological-associated diseases ${ }^{11}$, using in silico techniques and studying prominent residues in the active site. The authors calculated the binding energy of the five ligands and were able to identify the highest binding affinity with GB2, a component of Garcinia kolaviron, to the enzyme. Further, L. F. Calleja, et al. performed a docking analysis, via the UCSF Chimera package 1.5.2., to test ALDH1A1's interaction with a potential inhibitor, which showed that two potential orientations exist for omeprazole in the ALDH active site ${ }^{12,13}$. This study also reinforced the positive effect of using a large variety of substituents to calculate the activity within the active site. Computational studies with ALDH have also been studied using acetaminophen using the Swissmodel Server (http://swissmodel.expasy.org) for homology modeling, BSP-SLIM server (http://zhanglab.ccmb.med.umich.edu/BSP-SLIM/) for binding site calculations, and AutoDock Tools (ADT v1.4.2) and AutoDock Vina ${ }^{14}$ for binding studies. 


\section{Computational Methods:}

The crystal structure of the ALDH1A1 enzyme bound to the novel inhibitor CM026 was retrieved from the Protein Data Bank (PDB ID: 4WP7) ${ }^{10}$. The ALDH active site, which is defined as consisting of all amino acid residues with any atom within three angstroms of the bound ligand, was isolated and includes Ala462, Cys302, Gly125, Gly294, Gly458, His293, lle304, Phe171, Ser461, Thr129, Trp178, Tyr297, Val174, and Val460. All residues were capped with -OH or -H groups in order to maintain residue charge and polarity. These residues form a large and well defined active site (see Figure 4, for example).

The molecule suites that were tested in this study were designed based on previous work in this group $^{3,4,5}$. The LP-suite (Fig. 3.) was created based on the structure of dopamine-a natural ligand of the ALDH family--and most of these ligands carry a positive charge under physiological conditions. The LP molecules have a variety of substituents at the sixth position of the catecholic ring. These substituents are $\mathrm{X}=\mathrm{OH}, \mathrm{NO}_{2}, \mathrm{Br}, \mathrm{CN}, \mathrm{CHCH}_{2}$, and $\mathrm{COOH}$ as well as the cyclic variant. In previous studies, the $\mathrm{KH}$ and MP suites were created based on these LP molecules, but most of these carry a neutral charge (Fig. 3. ${ }^{4,5}$. The $\mathrm{KH}$ molecule tested in this study has a nitrile group in place of the amine tail of the LP molecules and a proton at the 6-position of the LP-suite. The neutral MP-suite was created by replacing the amine group of the LP-suite with a hydroxyl group. All suites were tested with similar substituents at the 6-position as the LP-suite. However, the cyclic variant is only present in the LP-suite and MP-suite. The LP-cyclic and MP-cyclic molecules place the amine tail or hydroxyl tail respectively into a heterocyclic ring 5 . 


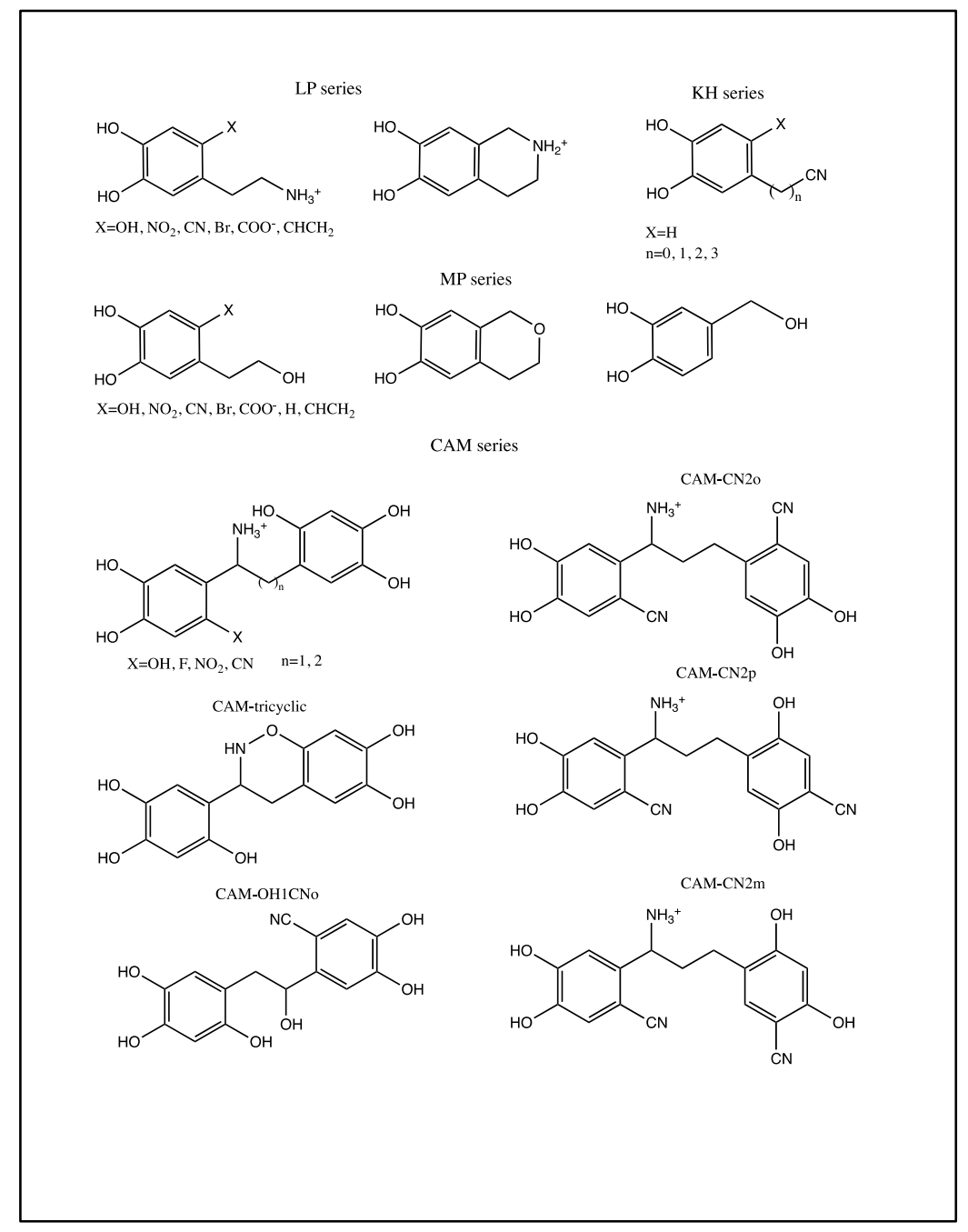

Figure 3. Ligands used for study.

Examining the smaller potential inhibitors and seeing how much empty space is between the inhibitors and the active site residues prompted a new study on larger ligands in the active site such as "2LP" molecular complexes and the CAM suite. The "2LP" molecular complexes are simply two LP molecules placed in the active site concurrently that were optimized at the same time. In all of the "2LP" optimizations carried out, the double inhibitors were run as if they were a singular compound but were not physically connected. Other than an increase in computational expense required, no additional 
challenges were encountered in optimizing these compounds. The orientation of the "2LP" molecular complex in the active site was studied to see how the binding energy was affected. Fig. 4A shows the "2LP" molecule in the A orientation with both of the $\mathrm{OH}$ substituents on the benzene ring near Trp178 in the active site. Fig. 4B shows the B orientation of both the LPs in the active site where the $\mathrm{OH}$ substituents are flipped toward the His293. Both Fig. 4A and Fig. 4B show pi-stacking with the "2LPs" in the active site. Fig. $4 \mathrm{C}$ shows orientation "O", the "opposite" orientation, where one LP molecule is in the A orientation and the second is in the B orientation. Seeing how the orientation and the size of the ligand had a positive effect on the binding energy led to the design of the CAM suite.

The CAM-suite is made up of double-ringed molecules and was created based on the "2LP" studies and the novel inhibitors $\mathrm{CM} 026$ and $\mathrm{CM} 053$ (Fig. 3). This key suite was designed to enhance the inhibitory performance for ALDH by developing a suite that behaves as two LPs linked together. Thus, we created a longer inhibitor that utilizes the space within the active site to make sure all residues were engaged. Having the double-ringed effect was implicated from the $\mathrm{CM} 026$ and $\mathrm{CM} 053$ inhibitors, as well as $\mathrm{CMO37,} \mathrm{which} \mathrm{has} \mathrm{a} \mathrm{tricyclic} \mathrm{structure.} \mathrm{This} \mathrm{suite} \mathrm{contains} \mathrm{various} \mathrm{substituents} \mathrm{and} \mathrm{the} \mathrm{option} \mathrm{of}$ extending, by adding carbon linkers between the two rings, or shortening the carbon chain (Fig. 3). Overall, the CAM-suite was created specifically for ALDH to accommodate the large active site and likely does not include a good candidate for binding to other enzymes in the dopamine pathway (Fig. 1).

The target of this study is to inhibit ALDH enzyme activity within the dopamine pathway, so all ligands tested are compared to the known inhibitors $\mathrm{CM} 026$, CM053, and $\mathrm{CM} 037$. In order for a ligand to be consider it must have a comparable interaction energy to the known inhibitor. Ligands tested in the ALDH enzyme that have promising inhibitory power can be tested in the proceeding and following enzymes in the dopaminergic pathway. 


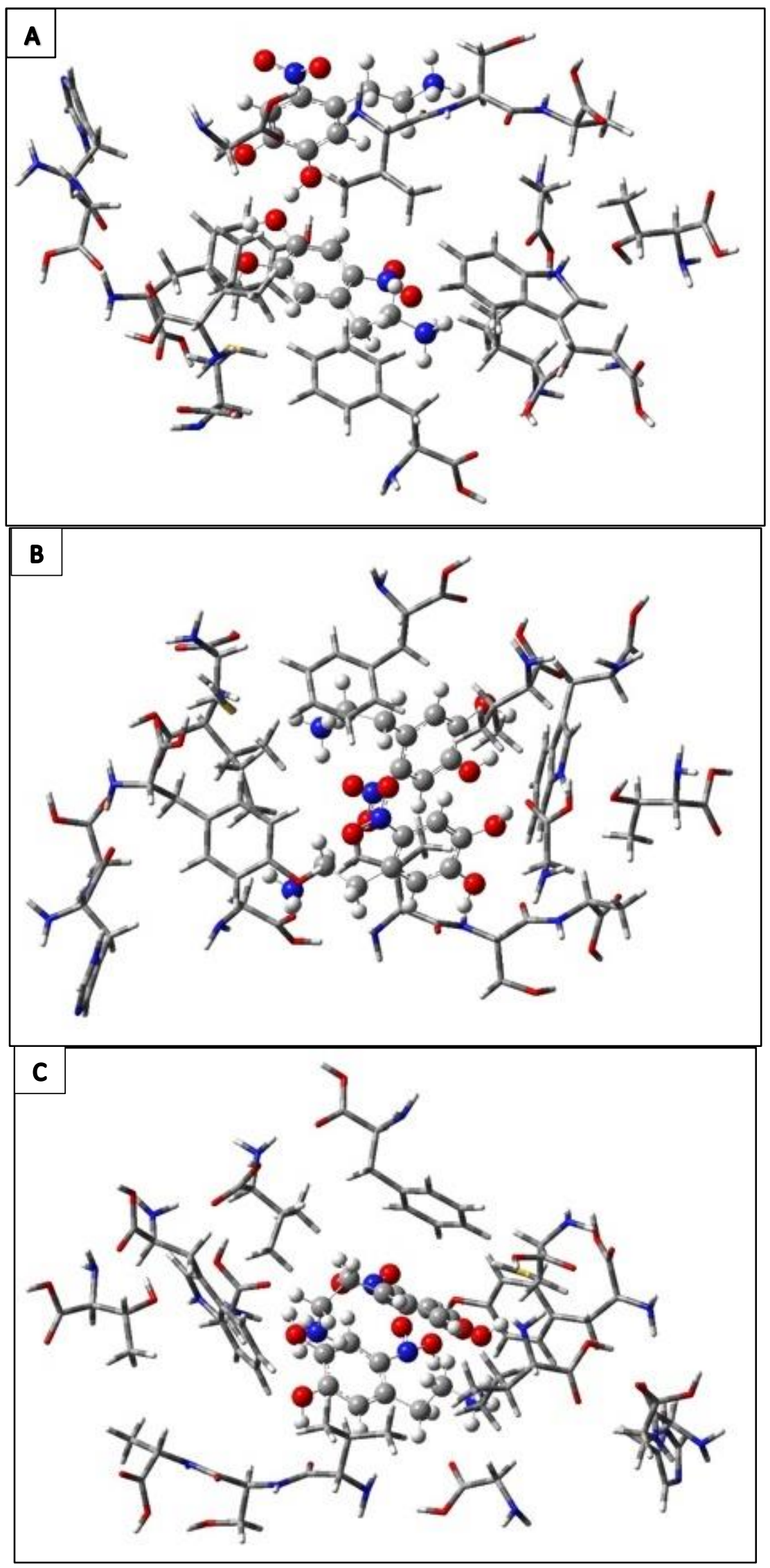


Figure 4. Molecular orientation of $2 L P-N O_{2}$ in the $A L D H$ active site. (A) $2 L P-N O_{2}$ in the " $A$ " orientation, (B) $2 L P-N O_{2}$ in the " $B$ " orientation, and (C) $2 \mathrm{LP}_{-} \mathrm{NO}_{2}$ in the " $\mathrm{O}$ " orientation.

The ligands were placed in the active site in the same basic starting configuration as $\mathrm{CM} 026$, which was the bound structure taken from the Protein Data Bank. Although the ligands, CM026, CM053, and CM037, used for comparison were structurally different, the inhibitors were positioned in the active site based on position of the ring structure and the polarity of the substituents. The catecholic ring within the ligands studied here was placed in the ALDH active site to mimic the $\mathrm{CM} 026$ and $\mathrm{CM} 053$ xanthine rings' position in the active site. Also, the more polar groups on the ligands' catechol group, such as the hydroxyl groups, were positioned corresponding to the more polar groups on the xanthine ring of CM's. The ligands with a carbon tail attached to a more polar substituent coming off the catecholic ring were positioned in the similar directionality as the more polar tail of the CM026 xanthine ring. The positions

of all ligands studied here were optimized in the active site using M06-2X ${ }^{15}$ and the basis set of 6-31G ${ }^{16}$. Relaxed amino acid residue side chains were implemented to allow for a general rigidity of the active site, but with flexibility in the side chains. Implicit solvation was used because previous studies have shown that the inclusion of solvation renders more realistic ion stability in the active site and correctly predicts protonation/deprotonation of amino acid side chains and ligands ${ }^{17}$. The dielectric constant used in this model was 78.3553 and the atomic radius used was from the UFF force field scaled by $1.1^{18}$. Once the ligand positions were optimized, the counterpoise-corrected energies for the ligand and each amino acid were calculated separately with MO62X and the $6-311+G *$ basis set and added together to obtain the total interaction energy. All calculations were done using Gaussian $16^{21}$.

\section{Results and Discussion:}

All of the interaction energy (IE) values are reported in $\mathrm{kcal} / \mathrm{mol}$. For the purposes of these results, the amino acid residues that most commonly had a strong positive or negative energy effect on the total 
interaction energy among the inhibitors tested were selected to be exhibited in the tables below (Ala462, Gly294, His293, Phe171, Ser461, Tyr297, Trp178, and Val460). The rest of the amino acid interaction energies, while they may have larger values for some ligands, are only included in the supplementary data file (Cys302, Gly125, Gly458, Ile304, Thr129, and Val174). All interaction energies were compared to the IEs for $\mathrm{CM} 026, \mathrm{CM} 053$, and $\mathrm{CM} 037$, known strong inhibitors for ALDH. In order to inhibit ALDH, an ideal ligand will have a stronger binding affinity (more negative number) for the active site than the known inhibitor. The eight amino acid residues shown here (Ala462, Gly294, His293, Phe171, Ser461, Tyr297, Trp178, and Val460) provide a range of some of the residues that had a strong attractive or repulsive effect on the total interaction energy of each inhibitor tested.

All of the molecules in the LP-suite had strong interactions, which were favorable in the ALDH active site. The neutral KH-molecules had weak interactions, which prompted the further study of the neutral molecules of the MP-suite. The MP-suite, neutral, was then tested, and all molecules of this suite also had weak interactions. The positively charged LP-suite was tested in two orientations within the active site - A and B, determining that orientation B had a stronger binding affinity. The positively charged "2LP"-suite was then tested and also had strong interactions. This prompted the study of the positively charged CAM-suite that had strong interactions in the ALDH active site, with an exception of one CAM molecule that is neutral.

\subsection{Known Inhibitors}

$\mathrm{CM} 026$, CM053, and CM037 were tested because they are known potent inhibitor of $\mathrm{ALDH}^{10}$

$\left(0.8,0.2\right.$, and $4.6 \mu \mathrm{m}$ relative $\mathrm{IC}_{50}$ values for $\left.\mathrm{ALDH} 1 \mathrm{~A} 1\right)$. These three inhibitors were our base line for testing and determining a new potential inhibitor that can surpass them. We calculated the IE's of each of the three known inhibitors shown in Table 1. Our calculated values correlate well to the experimental values, as the two compounds (CM026 and $\mathrm{CM} 053$ ) determined to have sub-micromolar potencies 
correspond to stronger interaction energies calculated here (-52 to $-76 \mathrm{kcal} / \mathrm{mol})$, while the less potent CM037 has an interaction energy less than half as strong as the other two $(22 \mathrm{kcal} / \mathrm{mol})$.

Table 1 M062X calculations of the interaction energies of the M062X optimized common ligand. Energies in $\mathrm{kcal} / \mathrm{mol}$.

\begin{tabular}{lcccccccc}
\hline & Phe171 & Tyr297 & Trp178 & Ala462 & Gly294 & His293 & Ser461 & Total \\
\hline CM026 & -2.31 & -12.81 & -20.26 & -4.59 & -3.93 & -1.51 & -1.22 & -76.94 \\
CM053 & -3.14 & -8.73 & -7.18 & -0.48 & -4.87 & -3.09 & 0.44 & -52.13 \\
CM037 & 0.1 & -9.77 & -0.63 & 0.004 & -1.25 & -0.33 & -0.36 & -22.38 \\
\hline
\end{tabular}

\subsection{LP Suite}

The first suite that was tested were the LP ligands. These ligands were derived from dopamine that contained an overall +1 charge. Overall the LP ligands performance ranged from $-12.9 \mathrm{kcal} / \mathrm{mol}$ to $36.9 \mathrm{kcal} / \mathrm{mol}$ throughout eleven of the LP series in the A orientation (Table 2a). These IEs show that the LP series would not be sufficient to act as inhibitors in ALDH's active site due to the weaker IE that was calculated by comparing it to $\mathrm{CMO} 26$ and $\mathrm{CM} 053$. $\mathrm{LP}-\mathrm{OH}, \mathrm{LP}-\mathrm{NO}_{2}$, and $\mathrm{LP}-\mathrm{CHCH}_{2}$ in the A orientation have the strongest total IEs, closest to the more promising model inhibitor, $\mathrm{CM} 026$. As for $\mathrm{LP}-\mathrm{COOH}$ in the A orientation, this inhibitor has a strikingly potent IE due to the interaction with Cys302 (IE $=-95.04$ $\mathrm{kcal} / \mathrm{mol}$, Supplementary Data). This strong interaction is due to the fact that the thiol group of the Cys orients itself close enough to the carboxyl group of the ligand (deprotonated under physiological conditions) that the proton from the thiol moves closer to the negatively charged carboxyl group, resulting in a negatively charged Cys residue and a positively charged LP-COOH ligand which interact via a coulomb force. For most of the ligands in the " $\mathrm{A}$ " orientation, the phenylalanine and histidine residues have consistently strong interaction energies with the ligands, indicating selectivity to target for drug design. 
As for the LP molecules in the "B" orientation, the total IE was noticeably stronger in comparison to the A orientation. The IE ranged from $-15.0 \mathrm{kcal} / \mathrm{mol}$ to $-53.0 \mathrm{kcal} / \mathrm{mol}$ in the " $\mathrm{B}$ " orientation (Table $2 \mathrm{~b}$ ). $\mathrm{LP}-\mathrm{OH}$, in the "B" orientation has the strongest total interaction energy, closest to the model inhibitors, $\mathrm{CM} 026$ and CM053. For the "B" orientation, Tyr297 and Val460 have very strong interaction energies, also indicating selectivity to target for drug design (Supplementary Data). Comparing the IE of the " $\mathrm{A}$ " to the " $\mathrm{B}$ " orientation, the " $\mathrm{B}$ " orientation is more favorable due to the stronger IEs that were produced.

Table 2a M062X calculations of the interaction energies of the M062X optimized LP-suite in the " $A$ " orientation. Energies in $\mathrm{kcal} / \mathrm{mol}$.

\begin{tabular}{lcccccccc}
\hline & Phe171 & Tyr297 & Trp178 & Ala462 & Gly294 & His293 & Ser461 & Total \\
\hline LP-OH & -7.63 & -4.27 & -0.58 & 0.72 & -3.96 & -7.85 & 0.15 & -32.3 \\
LP-Cyclic & -4.41 & -9.93 & -0.37 & 0.30 & -1.61 & -9.25 & 1.14 & -34.7 \\
LP-NO $_{2}$ & -8.25 & -3.83 & -0.36 & 0.69 & 0.13 & -10.0 & 1.20 & -36.1 \\
LP-Br & -11.25 & -5.49 & -2.63 & 0.33 & -1.76 & -8.85 & 0.22 & -29.9 \\
LP-CN & -11.30 & -4.61 & -1.29 & 0.90 & -2.58 & -8.47 & 1.07 & -36.9 \\
LP-CHCH & -2.54 & -1.99 & -1.05 & 0.86 & -3.21 & -7.02 & 0.74 & -29.1 \\
LP-COOH & -3.73 & -7.60 & -0.15 & 1.01 & -7.02 & -1.50 & 1.52 & -121.1 \\
\hline
\end{tabular}

Table $2 \mathbf{b}$ M062X calculations of the interaction energies of the M062X optimized LP suite in the "B" orientation. Energies in $\mathrm{kcal} / \mathrm{mol}$.

\begin{tabular}{lcccccccc}
\hline & Phe171 & Tyr297 & Trp178 & Ala462 & Gly294 & His293 & Ser461 & Total \\
\hline LP-OH & -0.42 & -17.28 & -2.31 & 0.45 & -1.58 & -0.46 & 1.83 & -53.0 \\
LP-Cyclic & -2.35 & -16.26 & -2.39 & 0.53 & -0.72 & 0.06 & -0.34 & -36.7 \\
$\mathrm{LP}_{-N O}$ & -1.67 & -9.24 & -2.12 & 0.49 & -1.59 & -0.40 & 1.81 & -48.0 \\
\hline
\end{tabular}




\begin{tabular}{lcccccccc}
\hline & Phe171 & Tyr297 & Trp178 & Ala462 & Gly294 & His293 & Ser461 & Total \\
\hline LP-Br & -1.80 & -7.95 & -2.47 & 0.45 & -1.65 & -0.45 & 0.45 & -47.2 \\
LP-CN & -0.91 & -9.79 & -2.14 & 0.50 & -1.51 & -0.37 & 1.66 & -47.5 \\
LP-CHCH & -2.92 & -9.35 & -2.91 & 0.42 & -1.64 & -0.44 & 1.46 & -48.4 \\
LP-COOH & -2.01 & -6.33 & -3.17 & 0.05 & -0.59 & -0.16 & 1.26 & -36.3 \\
\hline
\end{tabular}

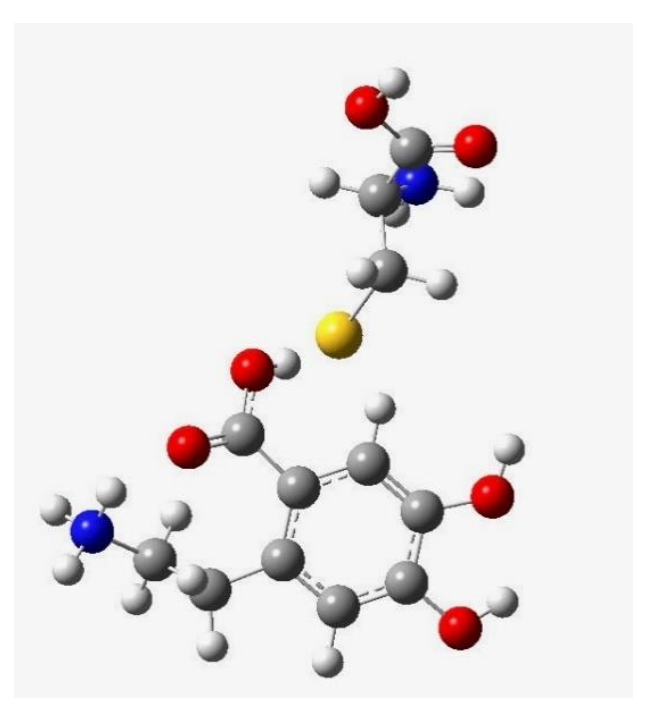

Figure 5. The ligand LP-COOH in the "A" orientation interacting with the CYS302 residue.

One of the major differences when comparing the novel inhibitors, CM026, CM053, and CM037, to the LP suite in both " $A$ " and " $B$ " orientation is in the size of these inhibitors. The large space within ALDH's active site may allow for larger inhibitors such as CM026 and CM053 to reach a stronger IE total. Thus, we studied cases where two LP molecules could bind to the active site at once, both in " $\mathrm{A}$ ", "B", and "O" (opposite) orientations.

Out of the "2LP" series, LP-NH2, LP-NO $2, \mathrm{LP}-\mathrm{Br}$, and LP-CN were each "double bound" in the ALDH active site and resulted in stronger interaction in the ALDH active site. This addition of the 2 LPs in 
the active site in each of the three orientations, " $\mathrm{A}$ ", " $\mathrm{B}$ ", and " $\mathrm{O}$ " (one ligand in $\mathrm{A}$ and the other in $\mathrm{B}$ ) orientation, produced a significantly stronger IE ranging from $-91.09 \mathrm{kcal} / \mathrm{mol}$ to $-141.97 \mathrm{kcal} / \mathrm{mol}$ (Table 3). Interestingly, the most competitive inhibitor from the "2LP" series was $2 \mathrm{LP}-\mathrm{Br} A$, which gave an IE of $-141.97 \mathrm{kcal} / \mathrm{mol}$ that wasn't consistent with the trend of the other IEs. This ligand has an electron withdrawing group $(-\mathrm{Br})$ that strongly interacted with $\mathrm{ALDH}$. The $2 \mathrm{LP}-\mathrm{Br} \mathrm{A}$ strongly interacted with Cys302 and Gly125, which became a major contributor to the overall IE total

Table 3 M062X calculations of the interaction energies of the M062X optimized 2-LP suite in the "A", "B", and "O" orientation. Energies in $\mathrm{kcal} / \mathrm{mol}$.

\begin{tabular}{|c|c|c|c|c|c|c|c|c|}
\hline & Phe171 & Tyr297 & Trp178 & Ala462 & Gly294 & His293 & Ser461 & Total \\
\hline $2 \mathrm{LP}-\mathrm{NH}_{2} \mathrm{~A}$ & -0.46 & -15.05 & -12.40 & -0.41 & -1.87 & -0.05 & 2.28 & -126.01 \\
\hline 2LP-NH ${ }_{2} B$ & -10.51 & -23.78 & -9.39 & 0.045 & -3.13 & -23.78 & 1.39 & -85.54 \\
\hline 2LP-NH ${ }_{2} \mathrm{O}$ & -8.33 & -19.73 & -3.41 & 0.44 & -2.97 & -0.81 & 0.94 & -104.97 \\
\hline $2 \mathrm{LP}-\mathrm{NO}_{2} \mathrm{~A}$ & -15.0 & -11.6 & -16.2 & 1.72 & -0.92 & -1.08 & 0.02 & -91.09 \\
\hline $2 \mathrm{LP}-\mathrm{NO}_{2} \mathrm{~B}$ & -21.4 & -21.4 & -18.3 & 1.30 & -0.63 & -1.06 & 2.15 & -135.77 \\
\hline $2 \mathrm{LP}-\mathrm{NO}_{2} \mathrm{O}$ & -12.9 & -20.2 & -18.5 & 2.11 & -0.78 & -1.35 & 2.29 & -124.30 \\
\hline 2LP-BrA & -40.1 & --17.1 & -19.8 & 3.07 & -1.24 & -0.79 & 1.65 & -141.97 \\
\hline 2LP-BrB & -19.2 & -22.2 & -12.6 & 1.25 & -0.92 & -1.46 & 3.52 & -131.31 \\
\hline 2LP-BrO & -8.58 & -9.74 & -19.4 & 2.29 & -0.65 & -1.08 & 1.65 & -113.61 \\
\hline 2LP-CNA & -16.0 & -18.1 & -17.7 & 2.58 & -1.34 & -0.97 & 1.52 & -116.57 \\
\hline 2LP-CNB & -21.2 & -17.6 & -12.5 & 1.17 & -0.81 & -0.84 & 2.04 & -122.86 \\
\hline 2LP-CNO & -10.5 & -18.7 & -20.6 & 2.48 & -0.58 & -0.95 & 1.18 & -129.60 \\
\hline $2 \mathrm{LP}-\mathrm{CHCH}_{2} \mathrm{~A}$ & - & - & - & - & - & - & - & - \\
\hline $2 \mathrm{LP}-\mathrm{CHCH}_{2} \mathrm{~B}$ & -24.5 & -9.32 & -15.0 & -0.97 & -1.00 & -1.21 & -3.49 & -115.27 \\
\hline $2 \mathrm{LP}-\mathrm{CHCH}_{2} \mathrm{O}$ & -12.7 & -15.2 & -24.1 & -1.53 & -1.23 & -1.04 & -1.72 & -118.91 \\
\hline
\end{tabular}




\subsection{KH-H molecule}

The $\mathrm{KH}$-suite was based on the LP-suite and involves the replacement of the charged amine tail for a neutral nitrile tail with various lengths of carbon chains and no substituent in the $6^{\text {th }}$ position. The overall charge of $\mathrm{KH}$-suite was neutral. In both the " $\mathrm{A}$ " and " $\mathrm{B}$ " orientation, the overall IE from both categories was shown to be in a range from $-12.9 \mathrm{kcal} / \mathrm{mol}$ to $-20.59 \mathrm{kcal} / \mathrm{mol}$ respectively (Table $4 a$ and 4b). These IEs were more positive than the LP ligands that were docked in the active site. As a result, these IEs were shown to not inhibit ALDH very effectively and the $\mathrm{KH}$ suite was not pursued further.

Table 4a M062X calculations of the interaction energies of the M062X optimized KH-molecule in the "A" orientation. Energies in $\mathrm{kcal} / \mathrm{mol}$.

\begin{tabular}{ccccccccc} 
& Phe171 & Tyr297 & Trp178 & Ala462 & Gly294 & His293 & Ser461 & Total \\
\hline $\mathrm{KH}-\mathrm{H}, \mathrm{n}=0$ & -2.03 & -4.58 & -0.06 & -0.02 & -1.61 & -6.97 & -0.07 & -19.7 \\
$\mathrm{KH}-\mathrm{H}, \mathrm{n}=1$ & -2.39 & -2.84 & 0.05 & 0.05 & 0.02 & -5.77 & 0.04 & -12.9 \\
$\mathrm{KH}-\mathrm{H}, \mathrm{n}=2$ & -4.45 & -4.33 & 0.02 & 0.03 & -2.45 & -1.62 & 0.09 & -18.1 \\
$\mathrm{KH}-\mathrm{H}, \mathrm{n}=3$ & -5.36 & -3.91 & -0.17 & 0.09 & -0.99 & -6.23 & 0.12 & -22.0
\end{tabular}

Table 4b M062X calculations of the interaction energies of the M062X optimized KH-molecule in the " $\mathrm{B}$ " orientation. Energies in $\mathrm{kcal} / \mathrm{mol}$.

\begin{tabular}{ccccccccc}
\hline & Phe171 & Tyr297 & Trp178 & Ala462 & Gly294 & His293 & Ser461 & Total \\
\hline $\mathrm{KH}-\mathrm{H}, \mathrm{n}=0$ & -1.69 & 0.45 & -2.15 & 0.14 & -0.01 & -0.01 & 0.73 & -14.9 \\
$\mathrm{KH}-\mathrm{H}, \mathrm{n}=1$ & -2.37 & -2.82 & -3.22 & 0.08 & 0.27 & 0.13 & 0.90 & -20.6 \\
$\mathrm{KH}-\mathrm{H}, \mathrm{n}=2$ & -4.24 & -2.46 & -3.71 & 0.06 & 0.27 & 0.11 & 0.94 & -21.3 \\
$\mathrm{KH}-\mathrm{H}, \mathrm{n}=3$ & -2.24 & -3.33 & -3.78 & 0.24 & 0.24 & 0.43 & 0.45 & -25.6 \\
\hline
\end{tabular}

\subsection{MP series}

The MP-suite was based on the LP-suite. This suite consisted of neutral ligands as compared to the previous suite that was charged. In this suite, the ammonium tail of the LP-suite was replaced with a neutral hydroxyl group. The IE of MP-OH, MP-Cyclic, and MP-NO $\mathrm{N}_{2}$ ligands were calculated. The MP suite 
IE's range from $-18.6 \mathrm{kcal} / \mathrm{mol}$ to $-24.1 \mathrm{kcal} / \mathrm{mol}$ (Table 5). These IE are significantly weaker energies than the single LP ligands, double LP ligands, and CAM suite. This results in dopamine being broken down because the inhibitor is not strong enough to inhibit ALDH.

Table 5 M062X calculations of the interaction energies of the M062X optimized MP suite. Energies in $\mathrm{kcal} / \mathrm{mol}$.

\begin{tabular}{lcccccccc}
\hline & Phe171 & Tyr297 & Trp178 & Ala462 & Gly294 & His293 & Ser461 & Total \\
\hline MP-OH & -3.29 & -3.34 & -0.78 & -0.09 & -2.85 & -5.75 & -0.10 & -24.1 \\
MP-cyclic & -0.64 & -6.43 & -0.18 & -0.01 & -0.52 & -7.25 & 0.03 & -18.6 \\
MP-NO $_{2}$ & -3.15 & -3.15 & -0.04 & 0.06 & 1.59 & -7.42 & 0.15 & -23.6 \\
\hline
\end{tabular}

\subsection{CAM series}

The CAM-suite was based on the double LP molecule suite in the ALDH active site as well as the known inhibitors, CM026. Unlike the other novel molecules studied here, the CAM molecules feature a double ring with a varied connector and different substituents on the rings (Fig. 3). The CAM molecules have the substituents $\mathrm{X}=\mathrm{OH}, \mathrm{F}, \mathrm{NO}_{2}$, and $\mathrm{CN}$, as well as $\mathrm{n}^{\text {th }}$ position that determines the number of carbon linkers added on with $n=1$ or 2 . The first CAM molecule shown in Figure 5 is CAM $-C N 2$, representing the $\mathrm{CN}$ substituent in the $\mathrm{X}$ position with two carbon linkers added. Figure 6 shows an example of the next set of three CAM molecules, all with two carbon linkers, with the nitrile varying in the ortho, para, and meta positions. After the identification of the carbon linkers, an "o", " $p$ ", or " $m$ " will follow the name indicating the nitriles' position on the adjacent ring (Fig. 3). In Figure 6, this image specifically shows CAM-CN2O, with the nitrile in the ortho position that replaced the $\mathrm{OH}$ substituent. Figure 5 demonstrates how important the proximity and interactions are between the residues and the CAM molecules resulting in a more promising collection of inhibitors. In Figure 5, it shows the major contacts of Phe 171, Trp 178, and Tyr 297 due to hydrogen bonding with CAM-CN2 (Table 6). The last 
positively charged CAM molecule is CAM-tricyclic (Fig. 3). The overall positive charge of the first 12 molecules of the CAM-suite indicates that they are potential competitive inhibitors for ALDH (Table 6). Lastly CAM-OH1CNo was added to the CAM-suite by reconstructing CAM-OH1 (Fig. 3). We removed the amine, added an $\mathrm{OH}$ substituent to the first carbon linker, and a $-\mathrm{CN}$ in the ortho position to further support the findings with the neutral molecules (Fig. 3). Attaching rings to the branches of the LP-suite would almost mimic the structure of the $\mathrm{CM} 026$ inhibitor since it is the more potent inhibitor out of the three novel inhibitors tested (CM026, CM053, and CM037). Every molecule of the CAM-suite had an IE that is significantly stronger than the IE of CM026, which suggests there should be in vitro inhibition of ALDH by these molecules.

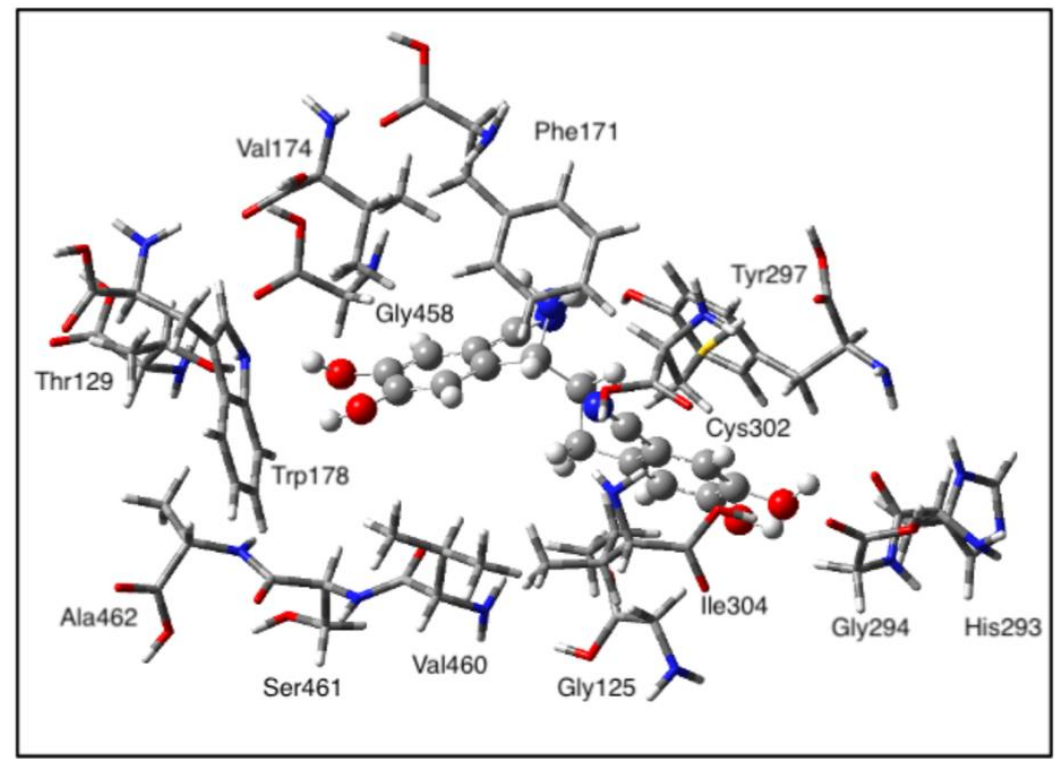

Figure 5. The inhibitor CAM-CN2 located in the ALDH active site with the labeled residues. 


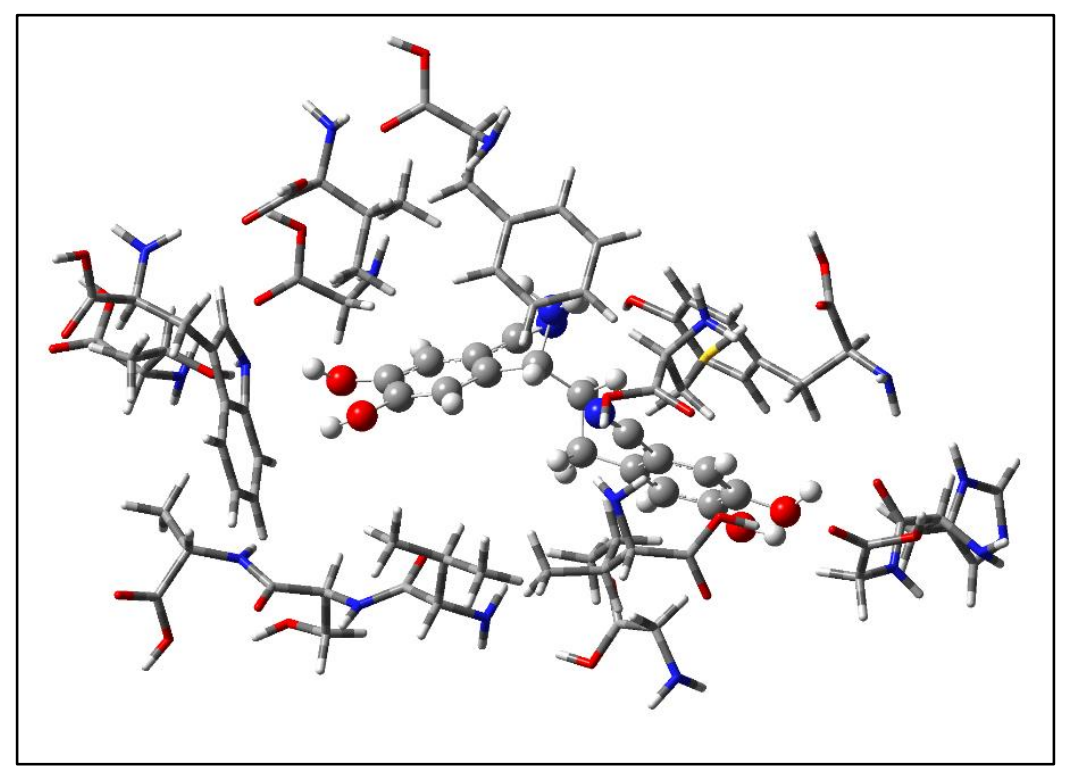

Figure 6. CAM-CN2O with two carbon linkers, and an additional nitrile substituting an $\mathrm{OH}$ substituent in the ortho director.

Table 6 M062X calculations of the interaction energies of the M062X optimized CAM suite. Energies in $\mathrm{kcal} / \mathrm{mol}$.

\begin{tabular}{lcccccccc}
\hline & Phe171 & Tyr297 & Trp178 & Ala462 & Gly294 & His293 & Ser461 & Total \\
\hline CAM-OH1 & -2.99 & -17.53 & -11.96 & 0.91 & -3.91 & -9.35 & 1.20 & -76.76 \\
CAM-F1 & -12.59 & -19.83 & -14.04 & 1.20 & -3.62 & -3.91 & 0.46 & -82.45 \\
CAM-NO 21 & -11.25 & -20.48 & -16.07 & 1.24 & -3.46 & -3.85 & 0.43 & -78.18 \\
CAM-CN1 & -11.5 & -23.02 & -14.91 & 1.15 & -3.46 & -4.10 & 0.38 & -87.54 \\
CAM-OH2 & -13.50 & -17.07 & -13.11 & 1.23 & -2.69 & -7.93 & 1.33 & -69.86 \\
CAM-F2 & -14.41 & -20.63 & -14.18 & 1.21 & -3.41 & -7.91 & 0.74 & -76.59 \\
CAM-NO22 & -14.21 & -20.14 & -16.22 & 1.32 & -2.62 & -0.54 & 0.36 & -79.42 \\
CAM-CN2 & -15.45 & -24.32 & -15.57 & 1.29 & -2.75 & -8.15 & 0.31 & -94.99 \\
CAM-CN20 & -18.70 & -22.33 & -15.09 & 1.27 & -1.96 & -9.30 & 0.45 & -86.51 \\
CAM-CN2p & -16.20 & -26.64 & -15.98 & 1.34 & -3.71 & -2.47 & 1.60 & -83.06 \\
CAM-CN2m & -0.44 & -25.55 & -15.86 & 1.35 & -5.16 & -9.53 & 0.86 & -76.44 \\
\hline
\end{tabular}




\begin{tabular}{lcccccccc}
\hline & Phe171 & Tyr297 & Trp178 & Ala462 & Gly294 & His293 & Ser461 & Total \\
\hline CAM-tricyclic & -4.39 & -6.81 & -9.09 & 0.28 & -2.55 & -1.01 & 0.01 & -37.25 \\
CAM-OH1CNo & -4.49 & -9.12 & -2.48 & -0.01 & -2.52 & -7.99 & 0.11 & -42.86 \\
\hline
\end{tabular}

\subsection{Structural support for interaction energy trends}

Overall, several interesting trends were observed for the different suites in ALDH. The positively charged LP-suite had strong interactions with the ALDH active site. Although LP-COOH is overall neutral, it has a strong positive and negative charge close to one another, so LP-COOH will still have the weak interactions within ALDH. In the LP-suite, all the molecules would lead to desired effects if they were made into PD drugs.

The neutral KH-molecules somewhat weakly interacted with the ALDH, which prompted us to test the MP-suite. The neutral MP-suite also had weak interactions with the ALDH active site. Both the KHmolecules and the MP-molecules strongly interacted with His and Tyr, which are consistently major contributors to the total interaction energies. The ligands that are neutral have tended to not be successful in inhibiting ALDH.

Interestingly, the orientation of the LP-suite in the ALDH active site, A or B, played an important role in discovering a slight difference in IE. The positive LP-suite in "B" orientation had stronger interactions than the " $A$ " orientation which was first studied. In the " $B$ " orientation, the $\mathrm{OH}$ groups off the benzene ring had stronger hydrogen bonding compared to the " $\mathrm{A}$ " orientation. The " $\mathrm{B}$ " orientation had much stronger hydrogen bonding with the residues, Gly125 and Val460. Anchoring down the ligand with the Tyr297 contributed to concluding that the " $\mathrm{B}$ " orientation is much more favorable in the ALDH active site. Orientation " $\mathrm{A}$ " lacks the additional intermolecular force of hydrogen bonding with the -OH tails. We can draw the conclusion that the " $\mathrm{B}$ " orientation, whose orientation is the opposite of the " $\mathrm{A}$ " orientation, had a strong influence on the IE. 
The positively charged " $2 \mathrm{LP}$ "-suite strongly interacted with the ALDH active site. Comparing this to the previously studied LP-suite, the double effect of the LPs in the active site created a strong potential inhibitor for ALDH. The individual two LPs in the active site were not bound together. The 2LP-suite strongly interacted with Phe171, Trp178, and Tyr297, which were major contributors to the total interaction energy. The orientations $A, B$, and $O$ gave mixed results when studying the 2LP-suite. Interesting, the result was that the double non-bound LP molecule within the active site doubled the IE therefore proving that ALDH requires a large inhibitor to effectively inhibit this enzyme.

Lastly, twelve out of thirteen ligands of the CAM-suite showed strong interactions in the active site. This is likely due to the $-\mathrm{NH}_{3}{ }^{+}$substituent. The molecules in the CAM-suite are larger and are designed to mimic a combination of $\mathrm{CMO26}$, the one of the known inhibitors tested, and the dopaminergic derivatives. The neutral CAM-OH1CNo molecule was tested and further indicated that the larger size alone was not enough toshow significant interaction. Interestingly, the positive CAM-tricyclic molecule with three rings showed an IE with weak bonding interactions. We have mentioned that the neutral ligands generally have weak interactions in the ALDH active site as shown by the KH-molecules and the MP-suite.

\section{Conclusion:}

If ALDH in the brain is inhibited by one of the novel ligands presented here, dopamine would be more slowly metabolized. This could increase the apparent effectiveness of L-DOPA therapy. It should be noted that an effective drug would need to overcome additional challenges posed by crossing the BBB. Current Parkinson's disease treatments such as L-DOPA and Carbidopa-both of which have charged moieties-are transported across the BBB via transporters. Current work in our group is examining the ability of proteins, such as Biogenic Amine Transporters, to move our potential inhibitors across membranes. Further, some of the most potent single molecule inhibitors reported here (CAM- 
$\mathrm{CN} 1, \mathrm{CAM}-\mathrm{CN} 2, \mathrm{CAM}-\mathrm{CN} 2 \mathrm{o}$ and CAM-CN2p) are neutral and thus more likely to cross membranes. The interaction energies shown here do not include entropic contributions, which could make the Gibbs energies differ from interaction energies. Previous work in our group has shown that for similar ligands in the active site, the relative numbers and trends obtained from interaction energies agree with those obtained from Gibbs energies ${ }^{20}$. Thus the comparisons obtained in this work should be consistent with experimental data (such as is shown for the CMOXX compounds). The only major difference should occur for the "2LP" type inhibitors, which, since they consist of two separate molecules, would lose considerably more entropy upon binding and their Gibbs energy trends would be shifted lower (less attractive) compared with the other molecule suites. Finally, previous work in our group shows how solvation/desolvation can affect the interaction energies calculated here ${ }^{22}$.

This study's work focuses on examining the interaction between the enzyme and the ligands created as well as the size, charge, and orientation of the ligand within the ALDH active site. The LP-suite was useful in determining the favored B orientation as well as knowing that we needed to test larger and more positive inhibitors. One of the most promising ligands we studied was $2 \mathrm{LP}-\mathrm{BrA}$ due to the electron withdrawing properties of the substituent - $\mathrm{Br}$ and the presence of " $2 \mathrm{LPs}$ " in the active site. In the $2 \mathrm{LP}$ study, the best orientation varied depending on the substituent. The larger, longer, and more positive ligands such as the CAM-suite have shown promising results for a potential inhibitor. Specifically, CAMCN2 which contains a net positive charge. Overall, larger and more positive ligands are favored to show inhibitory effects to ALDH to prolong L-DOPA. In future work we will synthesize and test the most promising ligands shown here.

\section{Acknowledgements}

This work was supported by the National Science Foundation (CHE-1626238). 


\section{References}

${ }^{1}$ I. Triarhou, Dopamine and Parkinson's disease. Madama Curie Bioscience Database, 2000-2013.

2 J. Dorszewska, et al., Molecular effects of L-dopa therapy in Parkinson's disease, Curr. Genomics 15 (2014) 11-17.

${ }^{3}$ R. Evans, L. Peterson, M. Cafiero, Evaluation of hybrid and pure DFT methods for the binding of novel ligands in tyrosine hydroxylase enzyme, Comput, Theor. Chem 1140 (2018) 145-151.

${ }^{4}$ A. K. Hatstat, M. Morris, L.W. Peterson, M. Cafiero, Ab Initio study of electronic interaction energies and desolvation energies for dopaminergic ligands in the catechol-O-methyltransferase active site, Comp, Theor. Chem 1078 (2016) 146-162.

${ }^{5}$ M. C. Perchik, L. Peterson, M. Cafiero, The effects of ligand deprotonation on the binding selectivity of phenylalanine hydroxylase active site, Comp, Theor. Chem 1153 (2019) 19-24.

${ }^{6}$ Fitzmaurice, Arthur $\mathrm{G}$ et al. "Aldehyde dehydrogenase inhibition as a pathogenic mechanism in Parkinson disease." Proceedings of the National Academy of Sciences of the United States of America. J. Med.Chem. 2012, 636-41. doi:10.1073/pnas.1220399110

${ }^{7}$ Y. Zhao, N.E. Schultz, D.G. Truhlar, Design of density functionals by combining the method of constraint satisfaction with parametrization for thermochemistry, thermochemical kinetics, and noncovalent interactions, J. Chem. Theory Comput. 2 (2) (2006) 364-382.

${ }^{8}$ Y. Zhao, D.G. Truhlar, Density functionals for noncovalent interaction energies of biological importance, J. Chem. Theory Comput. 3 (1) (2007) 289-300.

${ }^{9}$ E.G. Hohenstein et al., Assessment of the performance of the M05-2X and M06-2X exchange-correlation functionals for noncovalent interactions in biomolecules, J. Chem. Theory Comput. 4 (2008) 1996-2000.

${ }^{10}$ Morgan, C.; Hurley, T.; Characterization of Two Distinct Structural Classes of Selective Aldehyde Dehydrogenase 1 A1 Inhibitors. J. Med. Chem. 2015, 58, 1964-1975.

${ }^{11}$ T. H. Ogunwa, Computer-Aided Modeling of Interaction between Aldehyde Dehydrogenase and Garcinia Biflavonoids, Int. J. Comp. App. 179 (35) (2018) 18-35.

${ }^{12}$ L. F. Calleja, et al., Omeprazole as a potent activator of human cytosolic aldehyde dehydrogenase ALDH1A1, BBA - Gen. Sub. 1864 (2019) 129451.

${ }^{13}$ E.F. Pettersen, T.D. Goddard, C.C. Huang, G.S. Couch, D.M. Greenblatt, E.C. Meng, T.E. Ferrin, UCSF Chimera a visualization system for exploratory research and analysis, J. Comput. Chem. (13) (2004) 1605-1612.

${ }^{14} \mathrm{~A}$. O. Kolawole, Interaction of Aldehyde Dehydrogenase with acetaminophen as examined by spectroscopies and molecular docking, Biochem. Biophys. Rep. 10 (2017) 198-207.

${ }^{15}$ Y. Zhao, D.G. Truhlar, Theor. Chem. Acc. 120 (2007) 215-241.

${ }^{16}$ J.P. Blaudeau, M.P. McGrath, L.A. Curtiss, L. Radom, Extension of Gaussian-2 (G2) theory to molecules containing third-row atoms K and Ca, J. Chem. Phys. 107 (1997) 5016-5021.

${ }^{17}$ D. Bigler, L. Peterson, M. Cafiero, Effects of implicit solvent and relaxed amino acid side chains on the MP2 and DFT calculations of ligand-protein structure and electronic interaction energies of dopaminergic ligands in the SULT1A3 enzyme active site, Comput. Theor. Chem. 1051 (2014) 79-92.

${ }^{18}$ Gaussian 16, Revision B.01, M. J. Frisch, et al. Gaussian, Inc., Wallington CT, 2016

${ }^{22}$ A. K. Hatstat, M. Morris, L.W. Peterson and M. Cafiero, Ab initio study of electronic interaction energies and desolvation energies for dopaminergic ligands in the catechol-O-methyltransferase active site, Comput. Theor. Chem. Volume 1078 (2016) 146-162 